\title{
EDITORIAL
}

\author{
Jean-Marc Richard
}

\section{Special Issue on Critical Stability}

\author{
Preface
}

Published online: 28 August 2012

(C) Springer-Verlag 2012

This special issues contains 24 articles devoted to various aspects of Few-Body physics, under the title Critical Stability.

This is the physics of low-lying resonances, or weakly bound states, including improbable configurations whose stability sometimes challenges our naive intuition, and whose description requires new methods. The experimental approach to these states involves very sophisticated devices and analyses.

The applications are numerous and often of paramount importance. Historically, the negative hydrogen ion is perhaps the first halo: its stability requires quantum mechanics beyond a simple factorised wave function, and its existence is crucial in many chemical and biochemical processes, as well as for understanding the optical properties of the Sun's surface. Similarly, the nuclear synthesis uses nuclear states at the edge of stability.

The physics of critical stability is intrinsically interdisciplinary, with concepts and methods elaborated for nuclei, further developed for atoms, and vice-versa. Most contributors have themselves crossed several times the border of the physics of quarks, nuclei, atoms or molecules.

From these combined fields and from the variety of geographical and historical origins, this domain has inherited a rich vocabulary: universality, Efimov states, Borromean binding, Tango stability, rearrangement, etc., in addition to the already mentioned "halo", coming as a leitmotiv.

This physics is more and more discussed in atomic and nuclear-physics conferences, and of course in various "Few-Body" meetings. It has also motivated various dedicated workshops, where experts can discuss their progress and speculations in some more details. It is hoped that this special issue will inform both experts and non-experts about the recent developments.

I would like to thank the authors, the referees, and the editors of Few-Body Systems for the opportunity to publish this special issue, and Aksel Jensen, Alejandro Kievsky, André Martin and Antonino Zichichi for their help in gathering our colleagues and organizing talks and discussions that motivated this enterprise.

J.-M. Richard $(\varangle)$

Institut de Physique Nucléaire de Lyon, Université de Lyon

IN2P3-CNRS-UCBL, 4, rue Enrico Fermi,

69622 Villeurbanne, France

E-mail: jmrichar@ipnl.in2p3.fr 